\title{
Sociodemographic Factors Influenced Response to the 2015 National Nutrition Survey on Preschool Children: Results From Linkage With the Comprehensive Survey of Living Conditions
}

\author{
Hitomi Okubo and Tetsuji Yokoyama \\ Department of Health Promotion, National Institute of Public Health, Saitama, Japan \\ Received August 28, 2018; accepted January 3, 2019; released online March 2, 2019
}

\begin{abstract}
Background: The National Nutrition Survey on Preschool Children, Japan (NNSPC) provides fundamental information for policy making for child nutrition. However, the response rate and background characteristics of subjects are unclear. Here, we examined response rate and sociodemographic factors related with response to the survey and evaluated the magnitude of bias due to selective response in the survey estimates of the NNSPC.

Methods: This study was based on two national surveys conducted in 2015: the NNSPC and the Comprehensive Survey of Living Conditions (CSLC). Because potential survey participants of the NNSPC were children aged $<6$ years and their households that answered the CSLC, we examined response rates and respondent characteristics by linking the data of the NNSPC and CSLC. Multiple logistic regression analysis was used to identify sociodemographic factors associated with response. Potential bias caused by non-response in the survey estimates was examined after considering missingness through multiple imputation.

Results: Among the 5,343 children who participated in the CSLC, 3,426 children responded to the NNSPC (response rate $=64.1 \%$ ). Variables associated with response were living in a smaller city, a large number of children, three-generation family structure, older maternal age, and a non-working mother. The prevalence of overweight was underestimated by $20 \%$, but the bias for almost all variables examined was small.

Conclusions: Response to the survey varied by sociodemographic characteristics. Some biases, mostly small, were seen in survey estimates of the 2015 NNSPC. Further insight into the effect of selective response is important to assess associations between variables more precisely.
\end{abstract}

Key words: response rates; sociodemographic factors; multiple imputation; national surveys; Japan

Copyright $\odot 2019$ Hitomi Okubo et al. This is an open access article distributed under the terms of Creative Commons Attribution License, which permits unrestricted use, distribution, and reproduction in any medium, provided the original author and source are credited.

\section{INTRODUCTION}

It is now widely recognized that response rates in epidemiological studies have dramatically declined over the last few decades. ${ }^{1-3}$ Decreasing participation in survey research increases the risk of selection bias and errors in statistical inferences. ${ }^{4}$ Accumulating evidence of selection bias in health surveys has shown that participants differ from non-participants in several characteristics. ${ }^{5-16}$ In general, participants (respondents) are more likely to have a higher socioeconomic status (higher levels of education and income), ${ }^{5-12}$ a better health status, ${ }^{5,9}$ a healthier lifestyle, ,,9-13 and a lower mortality rate for specific diseases than nonparticipants (non-respondents). ${ }^{14-16}$

The National Nutrition Survey on Preschool Children (NNSPC), a questionnaire survey conducted in Japan every 10 years since 1985, assesses the feeding practices, dietary intake, lifestyle, and health status of preschool children living in Japan and provides valuable information for nutrition policy development. ${ }^{17}$ The survey findings have been used in the planning and promotion of breastfeeding and healthy diets for young Japanese children. However, the response rate and background characteristics of respondents, which are important indicators of representativeness, have not been published in any official reports. Survey participants of the NNSPC are drawn from among infants and preschool children (aged $<4$ years in 1985, 1995, and 2005, and aged $<6$ years in 2015) ${ }^{17}$ and their households who had answered another national survey, the Comprehensive Survey of Living Conditions (CSLC), ${ }^{18}$ conducted earlier in the same respective year. Linking data from the NNSPC and CSLC thus allows us to address response rates and the degree to which study participants differ from the total population. Understanding the background characteristics of respondents in the national survey is critically important for policy making and subsequent research. It might, therefore, be 
helpful to consider the possibility of selection bias and the external validity of the survey findings and to establish survey strategies for subject sampling and data collection.

The aim of the present study was to examine the response rate and sociodemographic factors related with response to the 2015 NNSPC by linking data with the CSLC. Additionally, we evaluated the magnitude of bias in the survey statistics obtained from the 2015 NNSPC due to selective response using a multiple imputation approach to account for mssingness. ${ }^{19}$

\section{METHODS}

\section{Data of two national surveys}

This study was based on data from two national surveys conducted in 2015 by the Japanese Ministry of Health, Labour and Welfare (MHLW): the CSLC ${ }^{20}$ and the NNSPC. ${ }^{21}$ Data from the two surveys were used with permission from the MHLW. The CSLC has collected comprehensive information on the living conditions of people in Japan, such as socio-demographics, health, medical care, welfare, and income, since 1986. The CSLC is conducted as a large-scale survey every 3 years and a smallscale survey in each interim year. ${ }^{18}$ The 2015 small-scale survey consisted of two surveys of household and income questionnaires. ${ }^{20}$ The household questionnaire covered 59,425 households and household members who lived in 1,106 districts randomly sampled from the National Census in 2010.22 The income questionnaire covered 9,036 and household members in 500 districts randomly selected from these 1,106 districts. The two questionnaires were distributed to potential eligible households in advance of each survey date (on June $4^{\text {th }}$ for the household questionnaire and July $16^{\text {th }}$ for the income questionnaire), and later collected by trained investigators during home visits. ${ }^{20}$ Of the 46,651 households that answered the household questionnaire (response rate $=78.5 \%),{ }^{20}$ data from 46,634 households $(115,941$ household members) for the household questionnaire and from 6,706 households (17,219 household members) for the income questionnaire were provided by the MHLW after excluding unclear answers.

The NNSPC has been conducted every 10 years since 1985 on the basis of the Statistics Act (General Statistical Surveys, Articles 19 to 23$)^{23}$ to assess methods of feeding in infancy and the diet and lifestyle of preschool children living in Japan and to obtain basic data required for the planning and promotion of breastfeeding and a healthy diet in early childhood. ${ }^{17}$ Potential participants of the NNSPC were children aged $<6$ years (born from 1st of June, 2009 to 31st of May, 2015) (approximately 5.5 thousand children) and their households (approximately 4.4 thousand children) in the 1,106 districts set for the 2015 CSLC. ${ }^{21}$ Of these, three districts in Ibaraki Prefecture were excluded from the survey in the aftermath of heavy rain which occurred in September 2015. Trained investigators visited each potential household a single time during September to distribute a selfadministered questionnaire for each child to the mother or guardian, who was usually responsible for food preparation, and later collected information about breastfeeding, weaning, introduction of solid foods, food allergy, child's health status and lifestyle, parental lifestyle, and basic characteristics of the family. Of the 2,992 households (3,936 children aged $<6$ years) who answered the questionnaire, ${ }^{21}$ data from 2,950 households $(3,871$ children) were provided by the MHLW after excluding those with unclear answers about child's age.
These surveys were conducted according to the guidelines of the Declaration of Helsinki, and verbal informed consent was obtained from all study participants or their guardian. The CSLC and NNSPC are both conducted by the MHLW, Japan, and have stringent protocols and procedures that ensure confidentiality and protect individual participants from being identified. Additionally, the present secondary analysis was based on a public-use dataset consisting solely of information that had already been anonymized. Accordingly, Institutional Review Board approval was not required.

\section{Data linkage}

As the potential study participants of the 2015 NNSPC were children aged $<6$ years and their households that answered the 2015 CSLC $^{20}$ we examined the characteristics of participants in the NNSPC by linking its data with those of the CSLC. We initially linked the two databases at the household level using information on prefecture, area, unit block, and household number that was common to both surveys. Among the 2,950 households of the 2015 NNSPC, 2,876 households (97.5\%) were linked to the 2015 CSLC. However, the 2015 CSLC answers of 80 of these 2,876 households revealed that they had no children aged $<6$ years, which meant that some data were linked incorrectly. In particular, $55.0 \%$ of these mislinked households were located in one prefecture. Careful review revealed that the household number assigned for the CSLC in this prefecture was not utilized in the NNSPC. To increase the accuracy of data linkage, we refrained from linkage at the household level and instead linked the two databases at the individual level.

Among the 115,941 participants of the 2015 CSLC, we initially restricted to the 5,343 children who were born from the 1st of June, 2009 to the 31st of May, 2015 (Figure 1). Information to identify each participant within the household was contained in the CSLC, but not in the NNSPC. Therefore, we linked the two databases at the individual level using the information on sex and birth year and month, in addition to the variables described above. Because there were some cases of multiple births (47 twins and 1 triplets), we also used information to identify multiple births within the households, which was newly created for the present study in consideration of birth order. For the one prefecture that did not use the same household number in both surveys, we used information on other variables apart from household number as an exception, and later confirmed that 60 individuals were additionally linked. Among the 5,788 children who answered either or both the CSLC and NNSPC, 1,917 children had data for the CSLC only, 445 children had data for the NNSPC only and 3,426 children had both. For the present study, we defined the 3,426 children who had both data and the 1,917 children who had data for the CSLC only as respondents and non-respondents to the NNSPC, respectively. Two authors independently conducted the data linkage and confirmed the consistency of the results.

\section{Assessment of sociodemographic and lifestyle characteristics}

Information on sociodemographic and socioeconomic variables was explored from the 2015 CSLC. $^{20}$ From the household questionnaire, we obtained information on child's sex (boy or girl), child's age as of May 31, 2015 (0-1, 2-3, and 4-5 years), household structure (parents and unmarried children only, single parent and unmarried children only, three-generation family, and 
The 2015 Comprehensive Survey of Living Conditions (CSLC)

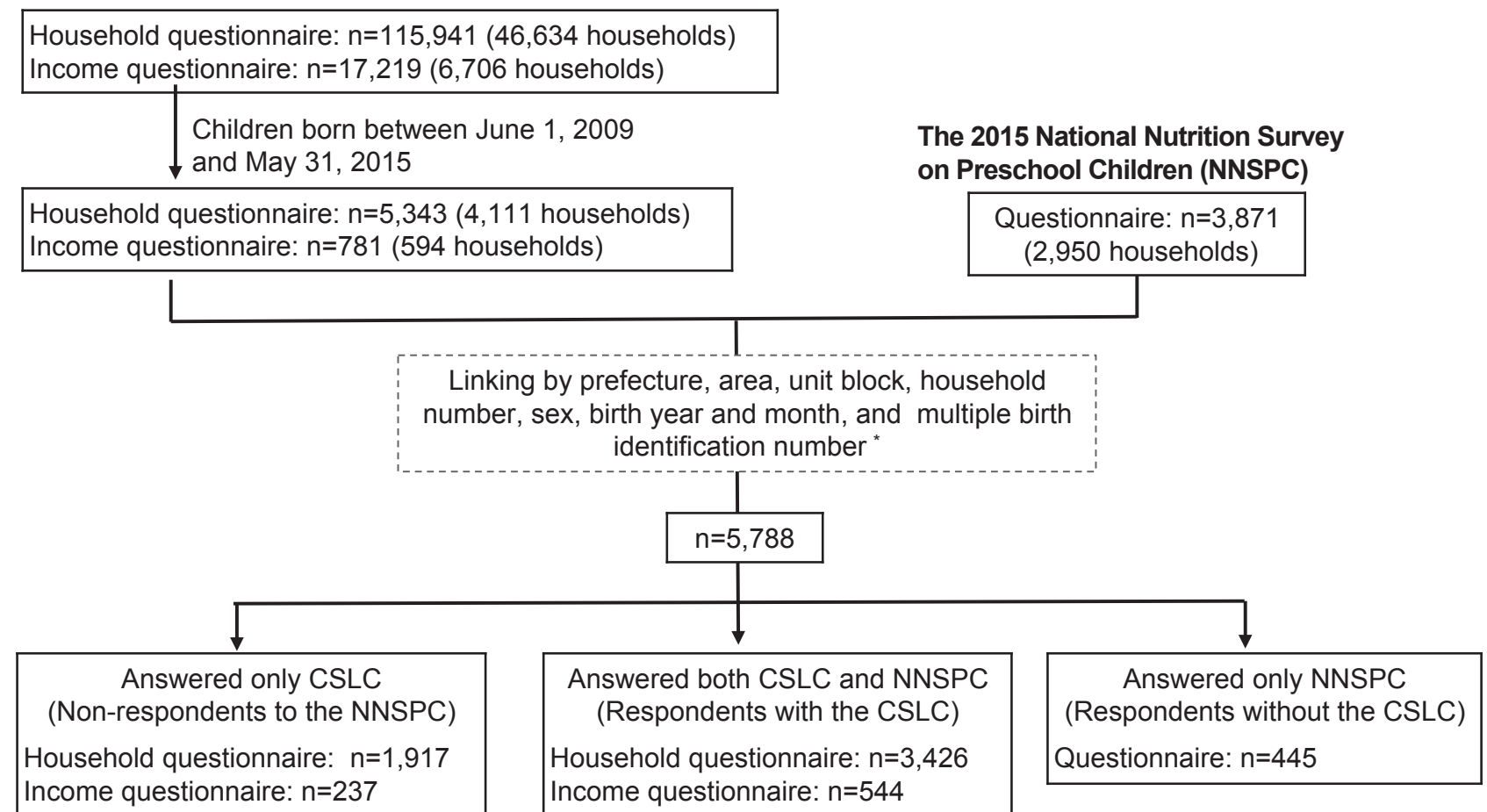

Figure 1. Flow chart of respondents in the 2015 NNSPC. ${ }^{a}$ One of 47 prefectures did not use the same household number in the CSLC and NNSPC. We therefore used information on other variables apart from household number for data linkage as an exception.

others), number of unmarried children aged $<18$ years $(1,2$, and $\geq 3$ persons), maternal and paternal educational attainment (junior high school or less; high school; technical or professional school/college; and university or higher), maternal and paternal labour force status (regular staff/employee; non-regular staff/ employee; executive of company/organization; self-employed/ family worker/industrial homework; others; and non-worker), and household expenditure in May. Equivalent household expenditure was calculated by dividing household expenditure in May by the square root of household $\operatorname{size}^{24}$ and then categorizing the result into thirds (low, middle, and high). Maternal and paternal age categories were defined $(<30,30-39$, and $\geq 40$ years). Residential blocks were grouped into six regions (Hokkaido and Tohoku; Kanto; Hokuriku and Tokai; Kinki; Chugoku and Shikoku; and Kyushu). Residential areas were also grouped into four categories according to population size (metropolitan areas; city with population $\geq 150$ thousand; city with population $<150$ thousand; and towns and villages; hereafter referred to as 'size of residential area'). Information on total household annual income and self-assessed living conditions (very difficult, somewhat difficult, normal, somewhat comfortable, and very comfortable) was obtained from the income questionnaire. Equivalent household annual income was calculated by dividing total household annual income by the square root of household size, and then categorizing the result into thirds (low, middle, and high). Missing data for household expenditure, maternal and paternal age, educational attainment, and labor force status were categorized as missing.

\section{Statistical analysis}

Response rates by sociodemographic variables were calculated using the number of participants aged $<6$ years in the 2015 CSLC as the denominator. The chi-square test was used to confirm homogeneity. Multivariate logistic regression analysis was performed, and the odds ratios (ORs) and 95\% confidence intervals (CIs) were calculated as measures of the strength of the association between the response and potential explanatory factor of interest. These analyses were controlled for potential confounders, including child's sex, child's age, size of residential area, residential block, household structure, number of unmarried children aged $<18$ years, equivalent monthly household expenditure, maternal age, maternal educational attainment, maternal occupation, paternal age, paternal educational attainment, and paternal occupation. Of the 4,111 households $(5,343$ children) included in the analysis, 1,117 households had at least two participating children. Therefore, we used robust standard errors to consider intraclass correlations among children in the same household. Some of the study participants answered the income questionnaire in the CSLC. We conducted a sub-analysis among subjects who answered the income questionnaire to examine the associations between economic characteristics (income level and self-assessed living conditions) and response to the NNSPC.

In the subsequent analysis, we examined the magnitude of potential bias in the survey estimates of the 2015 NNSPC due to missing data (non-response). There are two different sources of missing data in the NNSPC, the first attributable to respondents of the NNSPC who did not answer certain questions (item non-response) and the second to potential survey participants who did not respond to the 2015 NNSPC (survey non-response). We assumed that data were at least missing at random since the missing pattern was significantly determined by observed 
variables. ${ }^{19} \mathrm{We}$, therefore, used the multiple imputation procedure of the SAS statistical software (Proc MI; SAS Institute, Cary, NC, USA) to impute missing data and compared survey estimates of the NNSPC between data from respondents $(n=3,426)$ and those from potential survey participants $(n=5,343)$. Survey estimates selected here were child's sex, child's age as of May 31, 2015, birthweight and length, gestational age, current body weight and height, birth order, place of day care (eg, nurseries, kindergarten, certified centers for early childhood education and care, and so on), history of food allergy, bowel movements per week, wake-up time on weekdays, bedtime on weekdays, maternal age, maternal employment status, self-assessed economic condition, selfassessed time allowance, and self-assessed overall living conditions. These were selected because they were common question items observed in the 2015 NNSPC questionnaires for both 0-1year-old and 2-5-year-old children. To deal with item nonresponse among the respondents, the variables of the 2015 NNSPC mentioned above were used for the imputation model. The imputation process was repeated to create five imputed datasets using the fully conditional specification. For each of the five imputed datasets, we further imputed data for the 1,917 nonrespondents to the NNSPC to deal with survey non-response. The variables included in the imputation models were residential block, size of residential area, and household structure as well as the variables mentioned above. In total, 25 imputed datasets were created and the results were then averaged over these datasets using the MIANALYZE procedure of the SAS statistical software. To evaluate the magnitude of bias parameters due to missingness, we divided the difference in the survey estimates between respondents and potential survey participants after imputation by the imputed survey estimates in potential survey participants and multiplied by 100 . As the NNSPC data might be used to examine not only for point estimates of population parameters but also associations between variables, we need to know the magnitude of potential bias in associations between targeted outcomes and certain sociodemographic variables that significantly influence the response to the survey. We also examined ORs of overweight, as an example of targeted outcome, by sociodemographic variables before and after imputation. Overweight was defined according to the age- and sex-specific BMI cut-offs for Japanese children using the LMS method, which corresponded to BMI of $25 \mathrm{~kg} / \mathrm{m}^{2}$ at 17.5 years of age. ${ }^{25}$

All reported $P$ values are two-tailed, and $P<0.05$ was considered to be statistically significant. All statistical analyses were performed using SAS statistical software version 9.4.

\section{RESULTS}

\section{Response rate}

Of the 5,343 children aged $<6$ years who answered the household questionnaire of the 2015 CSLC, 3,426 children responded to the 2015 NNSPC. The overall response rate was $64.1 \%$.

Table 1 shows the response rate according to sociodemographic characteristics. Differences in response rate were observed across categories of almost all sociodemographic characteristics except for child's sex and age (all $P<0.05$ ).

\section{Association between sociodemographic factors and response to the 2015 NNSPC}

Table 1 also shows the multivariate odds ratios for response to the NNSPC. Potential sociodemographic predictors were size of residential area, residential block, household structure, number of children, maternal age, and maternal labor force status. Children who lived in a smaller city, especially in the Hokuriku, Tokai, and Kyushu areas, or lived in households with a large number of children were more likely to respond to the survey than the respective reference group. Three-generation households were more likely to respond to the survey than families of parents and unmarried children only. Among potential maternal characteristics, mothers who were older ( $\geq 40$ years) and who were nonworkers were more likely to respond to the survey. In contrast, no independent associations were observed for child's sex, child's age, equivalent household expenditure, maternal educational attainment, or any paternal variable examined in relation to response to the survey.

\section{Results from a sub-analysis using income question- naire}

Of the 781 children who answered the income questionnaire of the 2015 CSLC, 544 children (69.7\%) responded to the 2015 NNSPC. Table 2 shows the potential socioeconomic predictors of response among these responding subjects. Self-assessed living condition was significantly associated with response to the survey. Compared with households that assessed their living conditions as 'normal', those who assessed them as 'very difficult' were less likely to respond. This association remained after adjustment for potential confounders. On the other hand, we found no clear association between equivalent household annual income and response to the survey.

\section{Magnitude of bias due to selective response in survey estimates of the 2015 NNSPC}

Table 3 shows the magnitude of bias in survey estimates of the 2015 NNSPC, taking account of missing data (ie, item nonresponse and survey non-response) through multiple imputation. Survey estimates examined between respondents before and after imputation for item non-response (columns A and B, respectively) were not substantially different, which suggests that bias due to item non-response was quite small. When we compared survey estimates of respondents (B) and non-respondents (C) after imputation to examine the magnitude of bias attributable to survey non-response, prevalence of overweight was higher in non-respondents $(20.4 \%)$ than in respondents $(14.8 \%)$. This led to biased distribution in overweight, and prevalence of overweight among respondents (A) was underestimated by $20.3 \%$ in comparison with the imputed survey estimates among potential survey participants (D). In addition, the number of children receiving treatment for constipation was underestimated by $25.4 \%$ of respondents, while the number with an irregular bedtime was overestimated by $15.0 \%$ of respondents. However, these absolute differences were negligible $(-0.3 \%$ and $0.3 \%$, respectively). For all other variables examined, the bias was much smaller $(<5 \%)$.

To examine the potential bias in associations between targeted outcomes and certain sociodemographic variables that significantly influence the response to the survey, we provisionally compared ORs of overweight, as an example of targeted outcome, by sociodemographic variables before and after imputation (Table 4). The prevalence of overweight substantially changed in all categories of sociodemographic variables examined after imputation. The difference in OR of overweight before and after imputation was broadly observed. This tendency was more marked in certain categories with low response rate, such as 
Table 1. Response rates and odds ratios for response to the 2015 National Nutrition Survey on Preschool Children, Japan, according to sociodemographic characteristics among children aged $<6$ years and their households

\begin{tabular}{|c|c|c|c|c|c|c|}
\hline & \multirow{2}{*}{ Total } & \multicolumn{2}{|c|}{ Respondents } & \multirow{2}{*}{$P$ value $^{\mathrm{a}}$} & \multicolumn{2}{|c|}{ Multivariate $^{\mathrm{b}, \mathrm{c}}$} \\
\hline & & $n$ & Response rate, $\%$ & & OR & $95 \% \mathrm{CI}$ \\
\hline Number & 5,343 & 3,426 & 64.1 & & & \\
\hline \multicolumn{7}{|l|}{ Child's sex } \\
\hline Boys & 2,731 & 1,753 & 64.2 & \multirow[t]{2}{*}{0.92} & 1.00 & (Reference) \\
\hline Girls & 2,612 & 1,673 & 64.1 & & 0.96 & $(0.88-1.05)$ \\
\hline \multicolumn{7}{|l|}{ Child's age } \\
\hline $0-1$ year & 1,691 & 1,089 & 64.4 & \multirow[t]{3}{*}{0.94} & 1.00 & (Reference) \\
\hline $2-3$ years & 1,811 & 1,156 & 63.8 & & 1.00 & $(0.92-1.10)$ \\
\hline $4-5$ years & 1,841 & 1,181 & 64.1 & & 1.02 & $(0.91-1.13)$ \\
\hline \multicolumn{7}{|l|}{ Size of residential area } \\
\hline Metropolitan area & 1,537 & 877 & 57.1 & \multirow[t]{4}{*}{$<0.001$} & 1.00 & (Reference) \\
\hline City with population $\geq 150,000$ & 1,654 & 1,079 & 65.2 & & 1.40 & $(1.19-1.65)$ \\
\hline City with population $<150,000$ & 1,741 & 1,192 & 68.5 & & 1.56 & $(1.31-1.85)$ \\
\hline Towns and villages & 411 & 278 & 67.6 & & 1.47 & $(1.18-1.94)$ \\
\hline \multicolumn{7}{|l|}{ Residential blocks } \\
\hline Hokkaido and Tohoku & 488 & 295 & 60.5 & \multirow[t]{6}{*}{$<0.001$} & 1.00 & (Reference) \\
\hline Kanto & 1,940 & 1,185 & 61.1 & & 1.01 & $(0.80-1.27)$ \\
\hline Hokuriku and Tokai & 896 & 631 & 70.4 & & 1.40 & $(1.08-1.82)$ \\
\hline Kinki & 757 & 468 & 61.8 & & 1.04 & $(0.80-1.36)$ \\
\hline Chugoku and Shikoku & 522 & 346 & 66.3 & & 1.20 & $(0.90-1.61)$ \\
\hline Kyushu & 741 & 501 & 67.6 & & 1.39 & $(1.06-1.83)$ \\
\hline \multicolumn{7}{|l|}{ Household structure } \\
\hline Parents and unmarried children only & 4,326 & 2,778 & 64.2 & \multirow[t]{4}{*}{$<0.001$} & 1.00 & (Reference) \\
\hline Single parent and unmarried children only & 168 & 70 & 41.7 & & 0.64 & $(0.39-1.06)$ \\
\hline Three-generation family & 736 & 511 & 69.4 & & 1.35 & $(1.08-1.69)$ \\
\hline Others & 113 & 67 & 59.3 & & 1.02 & $(0.61-1.69)$ \\
\hline \multicolumn{7}{|l|}{ Number of unmarried children aged $<18$ years } \\
\hline 1 person & 1,659 & 984 & 59.3 & \multirow[t]{3}{*}{$<0.001$} & 1.00 & (Reference) \\
\hline 2 people & 2,429 & 1,589 & 65.4 & & 1.20 & $(1.04-1.40)$ \\
\hline$\geq 3$ people & 1,255 & 853 & 68.0 & & 1.24 & $(1.03-1.50)$ \\
\hline Equivalent household expenditure & & & & & & \\
\hline Low (<100,000 Japanese yen/month) & 1,632 & 1,049 & 64.3 & 0.01 & 1.00 & (Reference) \\
\hline Middle (100,000-139,999 Japanese yen/month) & 1,840 & 1,221 & 66.4 & & 1.09 & $(0.92-1.29)$ \\
\hline High $(\geq 140,000$ Japanese yen/month) & 1,770 & 1,101 & 62.2 & & 0.97 & $(0.82-1.15)$ \\
\hline Missing & 101 & 55 & 54.5 & & 0.69 & $(0.43-1.09)$ \\
\hline Maternal age & & & & & & \\
\hline$<30$ years & 1,037 & 624 & 60.2 & 0.02 & 1.00 & (Reference) \\
\hline $30-39$ years & 3,365 & 2,190 & 65.1 & & 1.20 & $(0.96-1.47)$ \\
\hline$\geq 40$ years & 908 & 594 & 65.4 & & 1.35 & $(1.03-1.77)$ \\
\hline Missing/children without mother & 33 & 18 & 54.5 & & 1.50 & $(0.40-5.61)$ \\
\hline Maternal educational attainment & & & & & & \\
\hline Junior high school or less & 206 & 121 & 58.7 & $<0.001$ & 1.00 & (Reference) \\
\hline High school & 1,636 & 1,035 & 63.3 & & 1.10 & $(0.77-1.56)$ \\
\hline Technical school or college & 1,868 & 1,280 & 68.5 & & 1.30 & $(0.91-1.87)$ \\
\hline University or beyond & 1,384 & 857 & 61.9 & & 1.05 & $(0.72-1.53)$ \\
\hline Missing/children without mother & 249 & 133 & 53.4 & & 1.08 & $(0.56-2.10)$ \\
\hline Maternal labor force status & & & & & & \\
\hline Regular staff/employee & 2,003 & 1,199 & 59.9 & $<0.001$ & 1.00 & (Reference) \\
\hline Non-regular staff/employee & 368 & 224 & 60.9 & & 1.05 & $(0.81-1.35)$ \\
\hline Executives of companies/organizations & 36 & 28 & 77.8 & & 2.28 & $(0.84-6.21)$ \\
\hline Self-employed/family worker/industrial homework & 329 & 232 & 70.5 & & 1.30 & $(0.95-1.77)$ \\
\hline Others & 38 & 25 & 65.8 & & 1.10 & $(0.51-2.34)$ \\
\hline Non-workers & 2,514 & 1,693 & 67.3 & & 1.47 & $(1.27-1.70)$ \\
\hline Missing/children without mother & 55 & 25 & 45.5 & & 0.70 & $(0.23-2.10)$ \\
\hline Paternal age & & & & & & \\
\hline$<30$ years & 664 & 419 & 63.1 & $<0.001$ & 1.00 & (Reference) \\
\hline $30-39$ years & 2,896 & 1,889 & 65.2 & & 0.95 & $(0.74-1.22)$ \\
\hline$\geq 40$ years & 1,436 & 944 & 65.7 & & 0.95 & $(0.71-1.26)$ \\
\hline Missing/children without father & 347 & 174 & 50.1 & & 1.35 & $(0.42-4.31)$ \\
\hline
\end{tabular}




\begin{tabular}{|c|c|c|c|c|c|c|}
\hline & \multirow{2}{*}{ Total } & \multicolumn{2}{|c|}{ Respondents } & \multirow{2}{*}{$P$ value $^{\mathrm{a}}$} & \multicolumn{2}{|c|}{ Multivariate $^{\mathrm{b}, \mathrm{c}}$} \\
\hline & & $n$ & Response rate, \% & & OR & $95 \% \mathrm{CI}$ \\
\hline \multicolumn{7}{|l|}{ Paternal educational attainment } \\
\hline Junior high school or less & 268 & 164 & 61.2 & $<0.001$ & 1.00 & (Reference) \\
\hline High school & 1,585 & 1,045 & 65.9 & & 1.08 & $(0.79-1.48)$ \\
\hline Technical school or college & 808 & 535 & 66.2 & & 1.15 & $(0.82-1.62)$ \\
\hline University or more & 2,146 & 1,408 & 65.6 & & 1.25 & $(0.90-1.74)$ \\
\hline Missing/children without father & 536 & 274 & 51.1 & & 0.77 & $(0.39-1.53)$ \\
\hline \multicolumn{7}{|l|}{ Paternal labor force status } \\
\hline Regular staff/employee & 4,112 & 2,665 & 64.8 & $<0.001$ & 1.00 & (Reference) \\
\hline Non-regular staff/employee & 73 & 44 & 60.3 & & 0.94 & $(0.57-1.55)$ \\
\hline Executives of companies/organizations & 227 & 155 & 68.3 & & 1.04 & $(0.73-1.48)$ \\
\hline Self-employed/family worker/industrial homework & 469 & 314 & 67.0 & & 1.04 & $(0.80-1.34)$ \\
\hline Others & 37 & 29 & 78.4 & & 1.49 & $(0.61-3.67)$ \\
\hline Non-workers & 65 & 40 & 61.5 & & 0.90 & $(0.50-1.59)$ \\
\hline Missing/children without father & 360 & 179 & 49.7 & & 0.79 & $(0.27-2.26)$ \\
\hline
\end{tabular}

CI, confidence interval; OR, odds ratio.

${ }^{a}$ The chi-square test was used to confirm homogeneity.

${ }^{\mathrm{b}}$ Considered intraclass correlations among children in the same household

${ }^{\mathrm{c}}$ Adjusted for all of the variables listed in the Table.

Table 2. Response rates and odds ratios for response to the 2015 National Nutrition Survey on Preschool Children, Japan, according to economic characteristics among children aged $<6$ years and their households who answered an income questionnaire

\begin{tabular}{|c|c|c|c|c|c|c|}
\hline & \multirow{2}{*}{ Total } & \multicolumn{2}{|c|}{ Respondents } & \multirow{2}{*}{$P$ value ${ }^{\mathrm{a}}$} & \multicolumn{2}{|c|}{ Multivariate $^{\mathrm{b}, \mathrm{c}}$} \\
\hline & & $n$ & Response rate, \% & & OR & $95 \%$ CI \\
\hline Number & 781 & 544 & 69.7 & & & \\
\hline \multicolumn{7}{|l|}{ Equivalent household annual income } \\
\hline Low $(<2,370,000$ Japanese yen $)$ & 260 & 173 & 66.5 & 0.38 & 1.00 & (Reference) \\
\hline Middle $(2,370,000-3,560,000$ Japanese yen $)$ & 261 & 188 & 72.0 & & 1.00 & $(0.63-1.58)$ \\
\hline High $(>3,560,000$ Japanese yen $)$ & 260 & 183 & 70.4 & & 0.84 & $(0.51-1.37)$ \\
\hline \multicolumn{7}{|l|}{ Self-assessed living conditions } \\
\hline Very difficult & 200 & 121 & 60.5 & 0.01 & 0.49 & $(0.30-0.79)$ \\
\hline Somewhat difficult & 276 & 196 & 71.0 & & 0.82 & $(0.53-1.26)$ \\
\hline Normal & 269 & 200 & 74.3 & & 1.00 & (Reference) \\
\hline Somewhat comfortable & 33 & 24 & 72.7 & & 100 & $(043-240)$ \\
\hline Very comfortable & 3 & 3 & 100.0 & & 1.02 & $(0.43-2.40)$ \\
\hline
\end{tabular}

CI, confidence interval; OR, odds ratio.

${ }^{a}$ The chi-square test was used to confirm homogeneity.

${ }^{\mathrm{b}}$ Considered intraclass correlations among children in the same household.

${ }^{\mathrm{c}}$ Adjusted for all of the variables listed in the Tables 1 and 2.

larger size of residential areas, single-parent families, and younger maternal age.

The statistics of the NNSPC published by the MHLW are generally estimated based on 3,871 NNSPC participants regardless of the availability of the CSLC data. Thus, we conducted the same analyses to examine the magnitude of bias in survey estimates among the NNSPC participants $(n=3,871)$ and the total survey participants of the CSLC and NNSPC $(n=5,788)$. The bias in point estimates for almost all variables, except for prevalence of overweight, in the NNSPC were small (eTable 1). Non-ignorable bias in ORs of overweight by sociodemographic variables was observed between before and after imputation (eTable 2).

\section{DISCUSSION}

By linkage to national data of the CSLC, we found that overall response rate of the $2015 \mathrm{NNSPC}$ was $64.1 \%$, and that response to the survey varied according to several sociodemographic characteristics of households. Response rates were substantially lower in metropolitan areas, as well as among families with a small number of children, households with difficulty in living conditions, and mothers who were younger and workers. Although some biases were seen in survey estimates of the NNSPC, the magnitude of bias due to this selective response was generally small.

To our knowledge, this is the first study to clarify the response rate and sociodemographic factors influencing the response to the NNSPC through linkage to the CSLC. Comparison with other studies is, therefore, limited to other national surveys that were sampled from the CSLC. For example, a few studies have examined response rates of the National Health and Nutrition Survey (NHNS), ${ }^{26}$ an annual nationwide survey which assesses the health status, food and nutrient intake, and lifestyles of people living in Japan, using linkage to the CSLC. ${ }^{27,28}$ Response rates to the questionnaire surveys of the NHNS from 2003 to 2007 were 
Table 3. Magnitude of bias in survey estimates among respondents of the 2015 National Nutrition Survey on Preschool Children, taking account of missingness through multiple imputation ${ }^{\mathrm{a}}$

\begin{tabular}{|c|c|c|c|c|c|}
\hline \multirow[b]{2}{*}{ Items obtained from the 2015 NNSPC } & \multirow[b]{2}{*}{$\begin{array}{c}\text { Respondents before imputation }{ }^{\mathrm{b}} \\
\text { (A) } \\
(n=3,426)\end{array}$} & \multicolumn{4}{|c|}{ Potential survey participants after imputation $^{\mathrm{c}}$} \\
\hline & & $\begin{array}{c}\text { Respondents } \\
\text { (B) } \\
(n=3,426)\end{array}$ & $\begin{array}{c}\text { Non-respondents } \\
\text { (C) } \\
(n=1,917)\end{array}$ & $\begin{array}{c}\text { All } \\
\text { (D) } \\
(n=5,343)\end{array}$ & Estimated bias, $\%^{\mathrm{d}}$ \\
\hline Child's sex, boy, \% & 51.2 & 51.2 & 51.0 & 51.1 & 0.1 \\
\hline \multicolumn{6}{|l|}{ Child's age, $\%$} \\
\hline $0-1$ year & 31.8 & 31.8 & 31.4 & 31.7 & 0.4 \\
\hline $2-3$ years & 33.7 & 33.7 & 34.2 & 33.9 & -0.4 \\
\hline 4-5 years & 34.5 & 34.5 & 34.4 & 34.5 & 0.0 \\
\hline Birthweight, g & 2998 & 2998 & 2991 & 2996 & 0.1 \\
\hline Birth length, $\mathrm{cm}$ & 48.8 & 48.8 & 48.8 & 48.8 & 0.1 \\
\hline Gestational week, wk & 38.7 & 38.7 & 38.7 & 38.7 & 0.0 \\
\hline Body weight, kg & 13.9 & 13.7 & 13.8 & 13.8 & 1.1 \\
\hline Body height, $\mathrm{cm}$ & 93.4 & 92.4 & 92.9 & 92.6 & 0.8 \\
\hline BMI, $\mathrm{kg} / \mathrm{m}^{2}$ & 15.8 & 15.9 & 15.8 & 15.9 & -0.1 \\
\hline Overweight, \% & 13.4 & 14.8 & 20.4 & 16.8 & -20.3 \\
\hline Birth order, first, \% & 45.6 & 45.6 & 45.9 & 45.7 & -0.2 \\
\hline \multicolumn{6}{|l|}{ Place of day care } \\
\hline Nurseries, yes, $\%$ & 34.8 & 34.8 & 34.5 & 34.7 & 0.4 \\
\hline Kindergarten, yes, $\%$ & 25.5 & 25.5 & 26.8 & 26.0 & -1.8 \\
\hline ECEC, yes, \% & 4.5 & 4.5 & 5.0 & 4.7 & -3.8 \\
\hline Home, yes, \% & 30.3 & 30.3 & 30.8 & 30.5 & -0.6 \\
\hline History of food allergy, $\%$ & 15.0 & 15.0 & 15.2 & 15.1 & -0.4 \\
\hline \multicolumn{6}{|l|}{ Bowel movements per week, $\%$} \\
\hline Almost everyday & 75.8 & 75.8 & 76.3 & 76.0 & -0.3 \\
\hline $4-5$ times/wk & 19.6 & 19.6 & 18.0 & 19.0 & 2.9 \\
\hline$\leq 3$ times/wk & 3.7 & 3.7 & 3.7 & 3.7 & -0.3 \\
\hline Under treatment & 1.0 & 1.0 & 2.0 & 1.3 & -25.4 \\
\hline \multicolumn{6}{|l|}{ Wake-up time on weekdays, $\%$} \\
\hline Before 7:00 & 46.5 & 46.6 & 45.9 & 46.3 & 0.5 \\
\hline 7:00-7:59 & 43.9 & 43.9 & 44.3 & 44.0 & -0.3 \\
\hline After 8:00 & 8.2 & 8.2 & 8.6 & 8.4 & -1.4 \\
\hline Irregular & 1.4 & 1.4 & 1.3 & 1.3 & 2.3 \\
\hline \multicolumn{6}{|l|}{ Bedtime on weekdays, $\%$} \\
\hline Before 21:00 & 28.3 & 28.3 & 29.4 & 28.7 & -1.5 \\
\hline 21:00-21:59 & 49.5 & 49.5 & 49.3 & 49.4 & 0.2 \\
\hline After 22:00 & 20.2 & 20.2 & 20.1 & 20.2 & 0.2 \\
\hline Irregularity & 2.0 & 2.0 & 1.3 & 1.7 & 15.0 \\
\hline \multicolumn{6}{|l|}{ Maternal age, $\%$} \\
\hline$<30$ years & 18.3 & 18.4 & 18.5 & 18.4 & -0.5 \\
\hline $30-39$ years & 64.2 & 64.2 & 62.7 & 63.6 & 0.9 \\
\hline$\geq 40$ years & 17.5 & 17.5 & 18.8 & 17.9 & -2.6 \\
\hline Maternal employment, yes, $\%$ & 49.7 & 49.9 & 47.3 & 49.0 & 1.5 \\
\hline \multicolumn{6}{|l|}{ Self-assessed economic condition, $\%$} \\
\hline Difficult & 37.5 & 37.5 & 38.0 & 37.7 & -0.5 \\
\hline Normal & 32.9 & 32.9 & 31.7 & 32.5 & 1.3 \\
\hline Comfortable & 29.6 & 29.7 & 30.2 & 29.9 & -0.8 \\
\hline \multicolumn{6}{|l|}{ Self-assessed time allowance, $\%$} \\
\hline Difficult & 47.2 & 47.2 & 46.8 & 47.0 & 0.3 \\
\hline Normal & 21.6 & 21.7 & 20.9 & 21.4 & 1.3 \\
\hline Comfortable & 31.2 & 31.2 & 32.3 & 31.6 & -1.3 \\
\hline \multicolumn{6}{|l|}{ Self-assessed overall living conditions, $\%$} \\
\hline Difficult & 20.8 & 20.8 & 23.5 & 21.7 & -4.5 \\
\hline Normal & 37.8 & 37.8 & 37.4 & 37.6 & 0.4 \\
\hline Comfortable & 41.5 & 41.5 & 39.1 & 40.6 & 2.0 \\
\hline
\end{tabular}

CSLC, the Comprehensive Survey of Living Conditions; ECEC, certified centers for early childhood education and care; NNSPC, the National Nutrition Survey on Preschool Children.

${ }^{\text {a }}$ Values are means for continuous variables and percentage for categorical variables.

${ }^{\mathrm{b}}$ Among 3,426 respondents to the NNSPC, data were missing for body weight $(n=265)$, body height $(n=378)$, BMI $(n=384)$, birthweight $(n=23)$, birth length $(n=64)$, gestational week $(n=60)$, place of day care $(n=7)$, history of food allergy $(n=21)$, bowel movements per week $(n=14)$, wake-up time $(n=12)$, bed time $(n=17)$, maternal age $(n=66)$, maternal employment $(n=80)$, self-assessed economic condition $(n=4)$, self-assessed time allowance $(n=2)$, and self-assessed overall living conditions $(n=2)$.

${ }^{c}$ Children aged $<6$ years and their households that answered the 2015 CSLC $(=3,426$ respondents $+1,917$ non-respondents).

${ }^{\mathrm{d}}$ Computed, before rounding, by dividing the difference in the survey estimates between the respondents (A) and potential survey participants after imputation (D) by the imputed survey estimates in potential survey participants (D) and multiplying by 100 .

${ }^{\mathrm{e}}$ Overweight was defined according to the age- and sex-specific BMI reference data for Japanese children using the LMS method that are corresponded to BMI of $25 \mathrm{~kg} / \mathrm{m}^{2}$ at 17.5 years of age. ${ }^{25}$ 
Table 4. Comparison of odds ratio of overweigh by sociodemographic characteristics estimated from observed and multiple imputation data

\begin{tabular}{|c|c|c|c|c|c|c|}
\hline & \multicolumn{3}{|c|}{ Observed data of the NNSPC respondents ${ }^{\mathrm{a}}$} & \multicolumn{3}{|c|}{ Multiple imputation data ${ }^{\mathrm{b}}$} \\
\hline & Prevalence, $\%^{\mathrm{c}}$ & $\mathrm{OR}^{\mathrm{d}}$ & $95 \% \mathrm{CI}$ & Prevalence, $\%^{\mathrm{c}}$ & $\mathrm{OR}^{\mathrm{d}}$ & $95 \% \mathrm{CI}$ \\
\hline \multicolumn{7}{|l|}{ Size of residential area } \\
\hline Metropolitan area & 11.2 & 1.00 & (Reference) & 15.5 & 1.00 & (Reference) \\
\hline City with population $\geq 150,000$ & 14.2 & 1.31 & $(0.98-1.75)$ & 17.2 & 1.12 & $(0.85-1.48)$ \\
\hline City with population $<150,000$ & 14.2 & 1.27 & $(0.95-1.70)$ & 17.6 & 1.17 & $(0.89-1.52)$ \\
\hline Towns and villages & 13.7 & 1.22 & $(0.79-1.89)$ & 16.2 & 1.05 & $(0.71-1.55)$ \\
\hline \multicolumn{7}{|l|}{ Household structure } \\
\hline Parents and unmarried children only & 13.1 & 1.00 & (Reference) & 16.6 & 1.00 & (Reference) \\
\hline Single parent and unmarried children only & 5.3 & 0.41 & $(0.12-1.32)$ & 13.3 & 0.93 & $(0.49-1.77)$ \\
\hline Three-generation family & 15.6 & 1.20 & $(0.90-1.61)$ & 18.7 & 1.19 & $(0.93-1.53)$ \\
\hline Others & 14.0 & 0.94 & $(0.42-2.11)$ & 17.8 & 1.00 & $(0.50,1.97)$ \\
\hline \multicolumn{7}{|l|}{ Maternal age } \\
\hline$<30$ years & 16.0 & 1.00 & (Reference) & 19.7 & 1.00 & (Reference) \\
\hline $30-39$ years & 12.4 & 0.83 & $(0.63-1.08)$ & 13.8 & 0.65 & $(0.55-0.78)$ \\
\hline$\geq 40$ years & 14.2 & 1.15 & $(0.81-1.64)$ & 14.9 & 0.90 & $(0.69-1.18)$ \\
\hline \multicolumn{7}{|l|}{ Maternal employment } \\
\hline Yes & 14.2 & 1.00 & (Reference) & 17.1 & 1.00 & (Reference) \\
\hline No & 12.5 & 0.77 & $(0.62-0.96)$ & 16.5 & 0.82 & $(0.68-0.99)$ \\
\hline
\end{tabular}

CI, confidence interval; CSLC, the Comprehensive Survey of Living Conditions; NNSPC, the National Nutrition Survey on Preschool Children; OR, odds ratio. ${ }^{a}$ Children aged $<6$ years and their households that answered both the 2015 CSLC and NNSPC $(n=3,426)$. Overweight was missing in 384 of the 3,426 respondents to the NNSPC in the observed data.

${ }^{\mathrm{b}}$ Children aged $<6$ years and their households that answered the $2015 \operatorname{CSLC}(n=5,343)$.

${ }^{\mathrm{c} O v e r w e i g h t ~ w a s ~ d e f i n e d ~ a c c o r d i n g ~ t o ~ t h e ~ a g e-~ a n d ~ s e x-s p e c i f i c ~ B M I ~ r e f e r e n c e ~ d a t a ~ f o r ~ J a p a n e s e ~ c h i l d r e n ~ u s i n g ~ t h e ~ L M S ~ m e t h o d ~ t h a t ~ a r e ~ c o r r e s p o n d e d ~ t o ~ B M I ~ o f ~}$ $25 \mathrm{~kg} / \mathrm{m}^{2}$ at 17.5 years of age. ${ }^{25}$

${ }^{\mathrm{d} A d j u s t e d ~ f o r ~ r e s i d e n t i a l ~ b l o c k s ~(H o k k a i d o ~ a n d ~ T o h o k u ; ~ K a n t o ; ~ H o k u r i k u ~ a n d ~ T o k a i ; ~ K i n k i ; ~ C h u g o k u ~ a n d ~ S h i k o k u ; ~ a n d ~ K y u s h u), ~ c h i l d ' s ~ s e x ~(b o y ~ o r ~ g i r l), ~}$ child's age (months), birthweight (g), birth length $(\mathrm{cm})$, and gestational week (wk).

$59.4-66.6 \%$ for the dietary survey and $59.2-67.9 \%$ for the lifestyle questionnaire. ${ }^{27} \mathrm{~A}$ similar but slightly lower response rate $(56.9 \%)$ was reported in the 2011 NHNS. ${ }^{28}$ Respondents of the NHNS were more likely to be women and older. ${ }^{27,28}$ In addition, response rates were higher in households with a large number of family members, three-generation families, and selfemployed families, and lower in single-parent families and employed families. ${ }^{27}$ In a comparison study with the Population Census in 2010, the NHNS sample had a lower proportion of individuals living in a metropolitan area (Kanto). ${ }^{29}$ Although direct comparison is hindered by differences in survey methods, target population, and survey year, the response rate of the NNSPC (64.1\%) was comparable to those for the NHNS and the results of this study are in accordance with the main tendencies of the findings of the NHNS: responses were associated with smaller cities, more family members, particularly children, a threegeneration family structure, and women (mothers) with older age. These findings from the NNSPC and NHNS suggest that residential area, number of household members, family structure, sex, and age are common predictors of a response to national surveys in Japan.

Partially consistent with previous studies, ${ }^{5-12}$ we observed that response to the survey was related with age and occupation. Of note, however, these relations were observed only in factors derived from mothers, not fathers. Because the questionnaires were answered mainly by mothers $(95.7 \%)$, response status might be more strongly affected by maternal factors. It is well-known that maternal socioeconomic factors and their health behaviors are associated with child's diet quality and general health behaviors. ${ }^{30-32}$ The respondents of the NNSPC might be already biased according to maternal characteristics. Although bias caused by selective response in ORs of overweight in the
NNSPC was observed, we still do not know the magnitude of bias in estimates of the other targeted outcomes across categories of maternal socioeconomic status. Further investigations of effects of bias by maternal and household sociodemographic characteristics is required to assess associations between variables more precisely. Moreover, any interpretation of survey findings on the relationships between maternal influence and child's diet require the closest attention.

The relation of some sociodemographic characteristics and response rates might be related to survey method. We speculated that a home-visit survey with a drop-off self-administered questionnaire as used in the NNSPC may involve more difficulty in contacting potential participants for survey invitation and/or data collection, particularly in areas with a large number of housing complexes, households with a small number of family members, and employees with long commutes, with consequent low response. Improving response rates therefore requires efforts to increase rates of contact with potential participants, and will in turn reduce selection bias. An increasing number of studies have investigated how to improve response rates in health surveys. ${ }^{33-36}$ Several studies examined possible reasons for non-participation in health surveys. Common answers among non-respondents were lack of time, unsuitable timing, increased number of survey requests, little personal benefit, and not considered relevant. ${ }^{33-35}$ A systematic review listed example strategies that might increase response to postal and electronic questionnaires, including monetary incentives, a more interesting questionnaire topic, prenotification, follow-up contact, and shorter questionnaires. ${ }^{36}$ Although the NNSPC uses a different survey method from the result of this systematic review, at least some of them would also likely be effective in increasing response rates in the NNSPC. Additional research on reasons for non-participation and ways to 
enhance motivation to participate in future surveys in Japan are warranted.

Missing data due to selected survey participation is a serious challenge and a threat to the validity of survey statistics. In the present study, we used a multiple imputation approach and conducted comparison between respondents, non-respondents, and potential survey participants after imputation to estimate magnitude of the bias in the survey estimates of the key variables. Although the response rate varied according to sociodemographic characteristics, bias in population estimates seemed quite small in most of the variables examined. In contrast, bias caused by selective response in prevalence and ORs of overweight was observed. These results suggest that the OR could be biased when the following two conditions are met simultaneously: 1) the prevalence of an outcome (eg, overweight) is substantially different between respondents and non-respondents, and 2) the response rate is substantially different between categories of a factor (eg, maternal age categories). An association analysis using data with low response rate should be carefully interpreted considering these conditions.

Among the strengths of our present study are its large nationwide sample of Japanese preschool children and their households from the general population. Further, linkage to the CSLC enabled us to extract a wide range of information on the sociodemographic and economic variables of households which we could then use to compare subject characteristics between respondents and non-respondents to the NNSPC. In addition, the use of the multiple imputation approach allowed us to maximize the available information through plausible replacement values for missing data and to evaluate the direction and magnitude of possible bias caused by selective response in survey estimates of the 2015 NNSPC.

Several limitations also need to be considered when interpreting the study findings. First, potential survey participants in the NNSPC (ie, respondents of the CSLC who were randomly sampled from the National Census in 2010) might already be inconsistent with the general population, given that the response rate of the 2015 CSLC was $78.5 \%{ }^{21}$ Second, we were limited in our comparison of sociodemographic factors between respondents and non-respondents. Although many studies have reported relationships among health status, lifestyle, and mortality, ${ }^{5,9-13}$ as well as of sociodemographic characteristics with response status, no such information was available in the CSLC we used because it was conducted as a small-scale survey in 2015. Third, there were 445 children who answered the NNSPC but were not linked with the CSLC, which in theory at least should not happen, considering that the CSLC provides sampling frames for the NNSPC. A few of these children were newly invited to the NNSPC because they had moved into the survey area during the survey period, and at least 236 children could not be linked to the CSLC because of inconsistent or missing information for one of the key variables used to identify individuals between the two surveys (ie, birth year, $n=50$; birth month, $n=21$; and sex, $n=165$ ). We do not know the exact proportion of these cases because of a lack of information and the expiration of the retention period of the originally answered questionnaires in the MHLW. In addition, subjects who had data for the NNSPC only were excluded from the present analysis, which might have led to further bias. This was done because some of the sociodemographic variables examined here were not available in the NNSPC, which made it difficult to compare the background characteristics of subjects with and without the CSLC data. Nevertheless, survey estimates of the 2015 NNSPC were not substantially affected regardless of the inclusion of NNSPC-only cases in multiple imputation. Fourth, the variables examined for the magnitude of bias were limited to common question items observed in questionnaires for 0-1-year-olds and 2-5-year-olds in the 2015NNSPC. Therefore, future studies aiming to more precisely assess diet-health relationships among young children should first confirm potential bias in estimates for other variables. Fifth, overweight, as an example of targeted outcome, was defined using BMI calculated from guardian-reported information, which might be biased. More importantly, BMI is based on only weight and height, both of which greatly change during growth and development. Finally, although we controlled for a wide range of potential confounders in multivariate logistic regression analysis, we cannot rule out the possibility of unmeasured or residual confounding, which is inherent to observational studies.

In conclusion, this study of a large sample of Japanese preschool children and their households showed that response to the survey varied according to sociodemographic characteristics, such as residential size and area, family structure, household economic status, and maternal age and labor force status. Allowing that certain subgroups are underrepresented, the general validity of survey estimates of the 2015 NNSPC, with the exception of prevalence of overweight, do not appear to be prejudiced by the bias produced by non-response. In addition to further improvement of response rate to the NNSPC, more insight into the effects of selective response is required to assess diet-health relationships among young children more precisely.

\section{ACKNOWLEDGEMENTS}

This study was funded by grants from the Japanese Ministry of Health, Labour and Welfare (H.O. \& T.Y., grant number H29sukoyaka-ippan-003). The Japanese Ministry of Health, Labour and Welfare had no role in the design, analysis, or writing of this article.

Conflicts of interest: None declared.

\section{APPENDIX A. SUPPLEMENTARY DATA}

Supplementary data related to this article can be found at https:// doi.org/10.2188/jea.JE20180176.

\section{REFERENCES}

1. Baruch Y, Holtom BC. Survey response rate levels and trends in organizational research. Hum Relat. 2009;61:1139-1160.

2. Mindell JS, Giampaoli S, Goesswald A, et al; HES Response Rate Group. Sample selection, recruitment and participation rates in health examination surveys in Europe-experience from seven national surveys. BMC Med Res Methodol. 2015;15:78.

3. NHANES Response Rates and Population Totals. https://wwwn. cdc.gov/nchs/nhanes/ResponseRates.aspx (Accessed October 8, 2018).

4. Meterko M, Restuccia JD, Stolzmann K, et al. Response rates, nonresponse bias, and data quality. Public Opin Q. 2015;79:130144.

5. Jackson R, Chambless LE, Yang K, et al. Differences between respondents and nonrespondents in a multicenter community-based study vary by gender ethnicity. The Atherosclerosis Risk in Communities (ARIC) Study Investigators. $J$ Clin Epidemiol. 
1996;49:1441-1446.

6. Tolonen H, Laatikainen T, Helakorpi S, Talala K, Martelin T, Prättälä R. Marital status, educational level and household income explain part of the excess mortality of survey non-respondents. Eur J Epidemiol. 2010;25:69-76.

7. Demarest S, Van der Heyden J, Charafeddine R, Tafforeau J, Van Oyen H, Van Hal G. Socio-economic differences in participation of households in a Belgian national health survey. Eur J Public Health. 2013;23:981-985.

8. Reinikainen J, Tolonen H, Borodulin K, et al. Participation rates by educational level have diverged during 25 years in Finnish health examination surveys. Eur J Public Health. 2018;28:237-243.

9. Hara M, Shimanoe C, Otsuka Y, et al. Factors associated with nonparticipation in a face-to-face second survey conducted 5 years after the baseline survey. J Epidemiol. 2015;25:117-125.

10. Turrell G, Patterson C, Oldenburg B, Gould T, Roy MA. The socioeconomic patterning of survey participation and non-response error in a multilevel study of food purchasing behaviour: area- and individual-level characteristics. Public Health Nutr. 2003;6:181189.

11. Strandhagen E, Berg C, Lissner L, et al. Selection bias in a population survey with register linkage: potential effect on socioeconomic gradient in cardiovascular risk. Eur J Epidemiol. 2010;25:163-172.

12. Iwasaki M, Otani T, Yamamoto S, et al; JPHC Study Group. Background characteristics of basic health examination participants: the JPHC Study Baseline Survey. J Epidemiol. 2003;13:216-225.

13. Hill A, Roberts J, Ewings P, Gunnell D. Non-response bias in a lifestyle survey. J Public Health Med. 1997;19:203-207.

14. Une H, Miyazaki M, Momose Y. Comparison of mortality between respondents and non-respondents in a mail survey. J Epidemiol. 2000;10:136-139.

15. Hara M, Sasaki S, Sobue T, Yamamoto S, Tsugane S. Comparison of cause-specific mortality between respondents and nonrespondents in a population-based prospective study: ten-year follow-up of JPHC Study Cohort I. Japan Public Health Center. J Clin Epidemiol. 2002;55:150-156.

16. Jousilahti P, Salomaa V, Kuulasmaa K, Niemelä M, Vartiainen E. Total and cause specific mortality among participants and nonparticipants of population based health surveys: a comprehensive follow up of 54372 Finnish men and women. $J$ Epidemiol Community Health. 2005;59:310-315.

17. Ministry of Health, Labour and Welfare, Japan. The National Nutrition Survey on Preschool Children (in Japanese). http://www. mhlw.go.jp/toukei/list/83-1a.html\#mokuteki (accessed April 2018).

18. Ministry of Health, Labour and Welfare, Japan. Comprehensive Survey of Living Conditions. http://www.mhlw.go.jp/english/ database/db-hss/cslc.html (accessed April 2018).

19. Sterne JA, White IR, Carlin JB, et al. Multiple imputation for missing data in epidemiological and clinical research: potential and pitfalls. BMJ. 2009;338:b2393.

20. Ministry of Health, Labour and Welfare, Japan. Comprehensive Survey of Living Conditions, 2015. (in Japanese) http://www.mhlw. go.jp/toukei/saikin/hw/k-tyosa/k-tyosa15/dl/01.pdf (accessed April 2018)

21. Ministry of Health, Labour and Welfare, Japan. The National Nutrition Survey on Preschool Children, 2015. (in Japanese). http://www.mhlw.go.jp/file/06-Seisakujouhou-
11900000-Koyoukintoujidoukateikyoku/0000134458.pdf (accessed April 2018).

22. Statistics Bureau, Ministry of Internal Affairs and Communications. Population and Households of Japan 2010: Overview of the Results of the 2010 Population Census. Tokyo: Statistics Bureau, Ministry of Internal Affairs and Communications; 2014.

23. Statistics Act. (Act No. 53 of May 23, 2007). http://www.soumu.go. jp/main_content/000463445.pdf (accessed April 2018).

24. Organisation for Economic Co-operation and Development. Growing Unequal? Income Distribution and Poverty in OECD Country. Paris: OECD; 2008.

25. Kato N, Takimoto $H$, Sudo N. The cubic functions for spline smoothed L, S and M values for BMI reference data of Japanese children. Clin Pediatr Endocrinol. 2011;20:47-49.

26. Ministry of Health, Labour and Welfare. National Health and Nutrition Survey. http://www.mhlw.go.jp/bunya/kenkou/kenkou eiyou_chousa.html (accessed May 2018).

27. Nishi N, Nakade M, Sarukura N, Nozue M, Tsubota M. Factors associated with response rates in National Health and Nutrition Survey. J Health Welfare Stat. 2012;59:10-15 (in Japanese).

28. Ando Y, Aoyama S, Ozaki T, Miura H, Yanagizawa T, Ishihama N. An analysis of response rates in National Dental Disease Survey: Almost all subjects of National Dental Disease Survey in 2011 were the subjects of blood test in the National Health and Nutrition Survey. Nihon Koshu Eisei Zasshi. 2016;63:319-324 (in Japanese).

29. Ikeda N, Takimoto H, Imai S, Miyachi M, Nishi N. Data Resource Profile: The Japan National Health and Nutrition Survey (NHNS). Int J Epidemiol. 2015;44:1842-1849.

30. Rogers I, Emmett P; ALSPAC Study Team. The effect of maternal smoking status, educational level and age on food and nutrient intakes in preschool children: results from the Avon Longitudinal Study of Parents and Children. Eur J Clin Nutr. 2003;57:854-864.

31. Robinson S, Marriott L, Poole J, et al; Southampton Women's Survey Study Group. Dietary patterns in infancy: the importance of maternal and family influences on feeding practice. Br J Nutr. 2007; 98:1029-1037.

32. Okubo H, Miyake Y, Sasaki S, Tanaka K, Murakami K, Hirota Y; Osaka Maternal and Child Health Study Group. Dietary patterns in infancy and their associations with maternal socio-economic and lifestyle factors among 758 Japanese mother-child pairs: the Osaka Maternal and Child Health Study. Matern Child Nutr. 2014;10:213225.

33. Chou P, Kuo HS, Chen CH, Lin HC. Characteristics of nonparticipants and reasons for non-participation in a population survey in Kin-Hu, Kinmen. Eur J Epidemiol. 1997;13:195-200.

34. Rönmark EP, Ekerljung L, Lötvall J, Torén K, Rönmark E, Lundbäck B. Large scale questionnaire survey on respiratory health in Sweden: effects of late- and non-response. Respir Med. 2009;103: 1807-1815.

35. Tolonen H, Lundqvist A, Jääskeläinen T, Koskinen S, Koponen $P$. Reasons for non-participation and ways to enhance participation in health examination surveys-the Health 2011 Survey. Eur J Public Health. 2017;27:909-911.

36. Edwards PJ, Roberts I, Clarke MJ, et al. Methods to increase response to postal and electronic questionnaires. Cochrane Database Syst Rev. 2009;(3):MR000008. 